\title{
99mTC-Methylene diphosphonate uptake at injury site correlates with osteoblast differentiation and mineralization during bone healing in mice
}

\author{
Zhendong A Zhong ${ }^{1,2}$, Anderson Peck ${ }^{3}$, Shihong $\mathrm{Li}^{3}$, Jeff VanOss ${ }^{3}$, John Snider ${ }^{1}$, Casey J Droscha ${ }^{1}$, Tingtung A Chang ${ }^{3}$ \\ and Bart O Williams ${ }^{1}$
}

${ }^{99} \mathrm{~m}$ Tc-Methylene diphosphonate $\left({ }^{99} \mathrm{~m}\right.$ Tc-MDP) is widely used in clinical settings to detect bone abnormalities. However, the mechanism of ${ }^{99 \mathrm{~m}}$ Tc-MDP uptake in bone is not well elucidated. In this study, we utilized a mouse tibia injury model, single-photon emission computed tomography (gamma scintigraphy or SPECT), ex vivo micro-computed tomography, and histology to monitor ${ }^{99} \mathrm{~m}$ Tc-MDP uptake in injury sites during skeletal healing. In an ex vivo culture system, calvarial cells were differentiated into osteoblasts with osteogenic medium, pulsed with ${ }^{99 \mathrm{~m}}$ Tc-MDP at different time points, and quantitated for ${ }^{99 \mathrm{~m}} \mathrm{Tc}-\mathrm{MDP}$ uptake with a gamma counter. We demonstrated that ${ }^{99 \mathrm{~m}} \mathrm{Tc}-\mathrm{MDP}$ uptake in the injury sites corresponded to osteoblast generation in those sites throughout the healing process. The ${ }^{99 \mathrm{~m}} \mathrm{Tc}-\mathrm{MDP}$ uptake within the injury sites peaked on day 7 post-injury, while the injury sites were occupied by mature osteoblasts also starting from day 7. ${ }^{99} \mathrm{~m}$ Tc-MDP uptake started to decrease 14 days post-surgery, when we observed the highest level of bony tissue in the injury sites. We also found that ${ }^{99 \mathrm{~m}} \mathrm{Tc}-\mathrm{MDP}$ uptake was associated with osteoblast maturation and mineralization in vitro. This study provides direct and biological evidence for ${ }^{99 \mathrm{~m}}$ Tc-MDP uptake in osteoblasts during bone healing in vivo and in vitro.

Bone Research (2015) 3, 15013; doi:10.1038/boneres.2015.13; Published online: 9 June 2015

\section{INTRODUCTION}

The treatment of severe bone diseases often requires invasive surgery; therefore, precise skeletal imaging is critical for efficient treatment. ${ }^{99 \mathrm{~m}} \mathrm{Tc}-M e t h y l e n e ~ d i p h o s p h o n a t e$ $\left({ }^{99 \mathrm{~m}} \mathrm{Tc}-\mathrm{MDP}\right)$ is extensively used as an imaging agent in single-photon emission computed tomography (SPECT). ${ }^{1-5}$ Though it is well known that ${ }^{99} \mathrm{~m}$ Tc-MDP accumulates in areas of active bone metabolism, the microscopic mechanism of ${ }^{99 \mathrm{~m}} \mathrm{Tc}-\mathrm{MDP}-$ bone uptake has not been well elucidated. To date, the ${ }^{99 \mathrm{~m}} \mathrm{Tc}-\mathrm{MDP}-u p t a k e$ mechanism has been investigated by (1) ex vivo studies measuring the uptake of ${ }^{99 \mathrm{~m}} \mathrm{Tc}-\mathrm{MDP}$ in synthesized bone-mimicking material, osteoblast-like cells, collagen sponges, or inorganic or organic phase of bone tissue and (2) in vivo imaging using bone-disease or bone-healing models with or without implants. The majority of previous work suggested that ${ }^{99} \mathrm{~m}$ Tc-MDP uptake was associated with mineralization, ${ }^{6-12}$ but some investigations argued that the organic compartment was also strongly involved. ${ }^{13-18}$

Most studies on the accumulation of ${ }^{99} \mathrm{~m}$ Tc-MDP in bone have lacked detailed information on osteoblast status; in some cases, the bone cells were dead when isotopebinding assays were performed. Osteoblast-lineage cells express different genes and behave differently throughout their differentiation/maturation from mesenchymal stem cells to mature osteoblasts and osteocytes. ${ }^{19}$ Osteoblasts are specialized cells that are able to secrete and mineralize the extracellular matrix (ECM), which is mainly composed of type I collagen along with smaller but significant, amounts of other proteins, including osteocalcin (Ocn). Once osteoblasts are embedded into bone matrix, they are defined as osteocytes. In response to skeletal injury, mesenchymal cells from the periosteum (a thin layer covering the bone outer surface) or other surrounding tissues invade the

'Center for Skeletal Disease Research, Van Andel Research Institute, Grand Rapids, MI, USA; ${ }^{2}$ Department of Internal Medicine, Center for Musculoskeletal Health, UC Davis Medical Center, Davis, CA, USA; ${ }^{3}$ Small Animal Imaging Facility, Van Andel Research Institute, Grand Rapids, MI, USA Correspondence: Tingtung A. Chang (anthony.chang@vai.org) and Bart O. Williams (Bart.Williams@vai.org) Received: 3 January 2015; Revised: 4 April 2015; Accepted: 21 April 2015 
wound site, proliferate, condense, differentiate into chondrocytes or osteoblasts, and mineralize. ${ }^{20}$

This study investigated the accumulation of ${ }^{99 \mathrm{~m}} \mathrm{Tc}-\mathrm{MDP}$ in bone in the context of osteoblast differentiation, using a skeletal injury model and a well-established reporter mouse model (Ocn-Cre:mT/mG) to mark mature osteoblasts.

\section{METHODS}

Ethics statement

All animal work was done in compliance with the guiding principles of the NRC's Guide to the Care and Use of Laboratory Animals. The protocol was approved by the Institutional Animal Care and Use Committee of the Van Andel Research Institute (animal protocol \#10-04-015). All surgeries were performed under anesthesia, and all efforts were made to minimize animal suffering.

\section{Mouse lines}

Ocn-Cre ${ }^{21}$ and $\mathrm{mT} / \mathrm{mG}^{22}$ transgenic mice were from Jackson Lab and kept in the Van Andel Institute Mutant Mouse Repository. The $\mathrm{mT} / \mathrm{mG}$ reporter mouse possesses loxp sites at both sides of a tdTomato (mT) cassette and expresses red fluorescence in all tissues. When bred to Creexpressing mice, the resulting offspring have the $\mathrm{mT}$ cassette deleted in the Cre-expressing tissues, allowing expression of the enhanced GFP ( $\mathrm{mG}$ ) cassette, which is located just downstream of $\mathrm{mT}$.

\section{Skeletal-injury surgery}

The detailed procedure was described previously. ${ }^{23}$ Briefly, three-month-old female Ocn-Cre;mT/mG mice were anesthetized with intraperitoneal injection of avertin. A 5-mm incision was made over the anteriorproximal tibia, and the tibial surface was exposed while carefully preserving the periosteum. A 1.0-mm hole was drilled through the anterior cortex with a high-speed dental drill. Wounds were closed with size 6-0 vicryl sutures. After surgery, mice received subcutaneous injections of tramadol for analgesia and were allowed to ambulate freely. Mice were scanned with SPECT/CT at indicated times post-surgery.

\section{${ }^{99 \mathrm{~m}} \mathrm{TC}-\mathrm{MDP}$ SPECT/CT}

Mice were placed in a $37.5^{\circ} \mathrm{C}$-heated cage $20-30 \mathrm{~min}$ utes prior to radiotracer injection and moved to a $37.5^{\circ} \mathrm{C}$-heated induction chamber 10 minutes prior to injection, where they were anesthetized with $2 \%$ isoflurane in $1000 \mathrm{cc} \cdot \mathrm{min}^{-1}$ oxygen. A dose of $1 \mathrm{mCi}$ in $0.1 \mathrm{~mL}$ of ${ }^{99 \mathrm{~m}} \mathrm{Tc}-\mathrm{MDP}$ was administered by tail vein injection and the mice were placed in a $37.5^{\circ} \mathrm{C}$-heated cage for 60 minutes of conscious uptake. SPECT/CT scans were acquired using a Bioscan NanoSPECT/CT (Washington,
DC) with fixtures to maintain heat and anesthesia throughout the process. Mice were imaged via a 24-minute static acquisition for SPECT imaging followed immediately by a 3-minute CT acquisition. SPECT reconstruction was performed using a dedicated Ordered Subset-Expectation Maximization algorithm and CT reconstruction used Filtered Back Projection with a Shepp-Logan Filter. Fused SPECT/CT images were analyzed using VivoQuant Image Analysis Suite (inviCRO, LLC, Boston, MA). The following standard operating procedures (SOP) were used: SOP 6.046 (SPECT imaging of mice); SOP 6.048 (CT imaging of mice).

\section{Osteoblast primary culture}

Calvarial cells were isolated from calvariae of newborn mice by serial digestion in $0.2 \mathrm{mg} \cdot \mathrm{mL}^{-1}$ collagenase type I (Worthington, Lakewood, NJ). Briefly, calvariae were digested for 15 minutes at $37^{\circ} \mathrm{C}$ with constant agitation. The digestion solution was collected and digested for additional five times. Digestions 3-6 (containing the osteoblasts) were centrifuged, washed with $\alpha$-MEM containing $10 \%$ fetal bovine serum and $1 \%$ penicillin/streptomycin, and cultured for 48 hours at $37^{\circ} \mathrm{C} .^{20}$ The same numbers of calvarial cells were cultured in 35-mm dishes and differentiated into osteoblasts with osteogenic media (change every 2 days) containing $50 \mu \mathrm{g} \cdot \mathrm{mL}^{-1}$ ascorbic acid and $10 \mathrm{mmol} \cdot \mathrm{L}^{-1} \beta$-glycerol phosphate ( $\beta G P$ ).

In-vitro ${ }^{99 m}$ Tc-MDP analysis

The osteoblasts in 35-mm dishes (three plates for each group) were incubated in $100 \mu \mathrm{L}$ of $0.9 \%$ saline diluted ${ }^{99 \mathrm{~m}} \mathrm{Tc}-\mathrm{MDP}\left(3.7 \mathrm{mBq}\right.$ ) for exact 40 minutes at $37^{\circ} \mathrm{C}$ and $5 \%$ $\mathrm{CO}_{2}$ atmosphere. The medium was aspirated, and then the dish was twice washed gently with $0.45 \mathrm{~mL}$ ice-cold $0.9 \%$ saline. The cells in each dish were digested with 0.2 $\mathrm{mL} 1 \mathrm{X}$ trypsin-EDTA for 15 minutes. The suspension of cell clusters was pipetted cautiously. The dishes were further washed twice with $0.9 \mathrm{~mL}$ of $0.9 \%$ saline. The cell suspension was combined with the washing saline. The residual mineral component in dish was washed with $2 \mathrm{~mL} 100$ $\mathrm{mmol} \cdot \mathrm{L}^{-1} \mathrm{HCl}$ for 15 minutes and the solution was collected. Planar gamma camera images were acquired for the dishes before and after aspirating the medium, and also following the cell removal and acid washing steps, respectively. A ${ }^{99 \mathrm{~m}} \mathrm{Tc}$ standard was measured simultaneously with the samples. The percentage of ${ }^{99 \mathrm{~m}} \mathrm{Tc}$ radioactivity in the cell culture (the cells and the cellexcreted mineral) was calculated based on the gamma imaging data. The ${ }^{99 \mathrm{~m}} \mathrm{Tc}$ radioactivity in the collected medium, digested cell suspension, and $\mathrm{HCl}$ samples

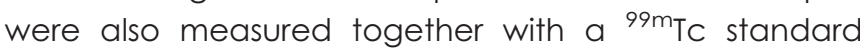
using a gamma counter (Perkin Elmer "Wizard 3" 2480 Automatic Gamma Counter). The percentage 
of ${ }^{99} \mathrm{~m}$ Tc-MDP in cells and residual mineral fraction was calculated after decay time adjustment.

Immunohistochemistry and staining

Bone tissue samples were fixed in $4 \%$ paraformaldehyde overnight and decalcified in 10\% EDTA for 10 days. Tissues were paraffin-embedded, and 7- $\mu \mathrm{m}$ sections were adhered to glass slides. Anti-GFP IHC was performed with Ventana Discovery Ultra (Tucson, AR). TRAP staining was performed using a leukocyte acid phosphate kit (Sigma) with fast red violet.

Quantitative real-time RT-PCR analysis

Total RNA was extracted from cells using RNeasy (Qiagen). The extracted RNA was used for CDNA synthesis via reverse transcriptase using Superscript III and random primers (Invitrogen). The cDNA samples were subjected to PCR analysis using Taqman PCR Master Mix and $\times 20$ mouse axin2 primer and probes (Applied Biosystems). Amplifications were then performed on an ABI 7500 Real-time PCR system. The expression of the gene of interest and the housekeeping gene (18S ribosomal RNA) were simultaneously determined in the same sample. For each sample, mRNA levels for each gene were normalized to 185 ribosomal RNA levels.

Statistical analyses

Results are presented as mean $\pm S D$, with $n$ equal to the number of samples analyzed. Student's t-test was used to test for significant differences between data sets.
Significance was defined as $P<0.05$, and all statistical analyses were performed with Microsoft Excel.

\section{RESULTS}

Skeletal injury model

To study the relationship between osteoblast maturation

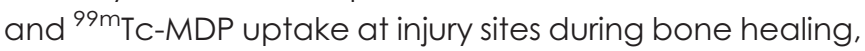
we used a mouse reporter strain (Ocn-Cre;mT/mG) that possesses both Cre recombinase driven by an osteoblast-specific osteocalcin promoter (Ocn-Cre) and a reporter transgene $\mathrm{mT} / \mathrm{mG}$ (Figure 1a). Ocn-Cre;mT/mG mice express green fluorescent protein (GFP) in mature osteoblasts and osteocytes. ${ }^{22,24}$ We traced osteoblast differentiation by using immunohistochemical (IHC) analysis with anti-GFP antibody in dissected bone tissue (Figure 1b). For the skeletal injury model, a 1-mm hole was made in the proximal tibia with a dental drill (Figure 1c). At the indicated time points post-surgery, the animals were evaluated by SPECT/computed tomography (CT) scan 1 hour after intravenous ${ }^{99 \mathrm{~m} T \mathrm{C}-M D P}$ injections. The tibia samples were collected from 3 to 5 mice at each time point for an ex vivo micro-computed tomography (microCT) scan (Figure 1c) and histological analysis (Figure 1d).

\section{${ }^{99 \mathrm{~m}}$ Tc-MDP-uptake dynamics during bone healing correlate with osteoblast generation in injury sites} We monitored the bone healing using ex-vivo micro-CT at each time point, and found that mineralized mass started a

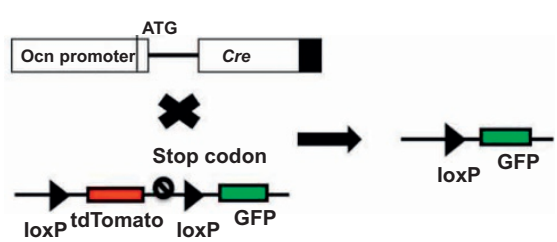

C

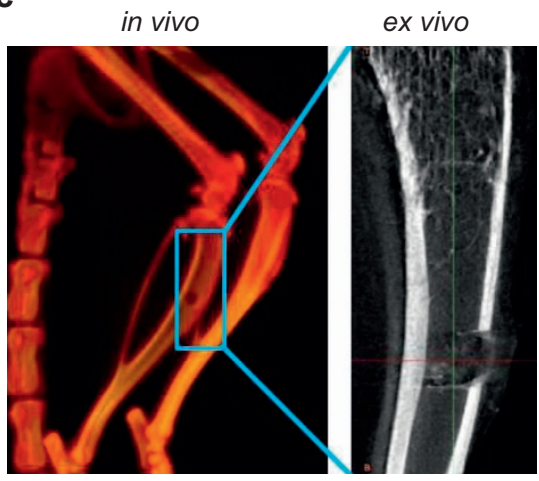

b

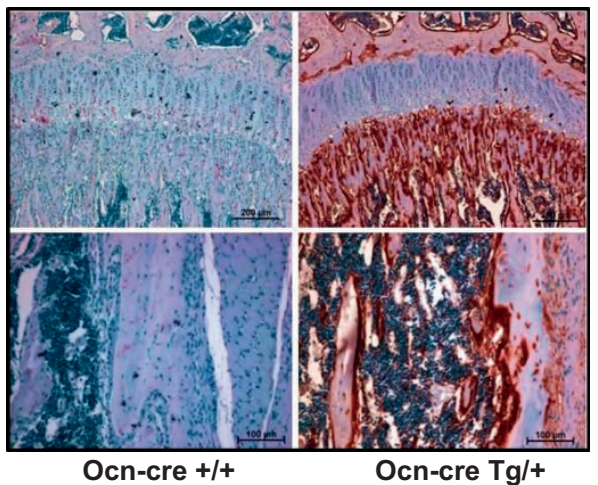

d

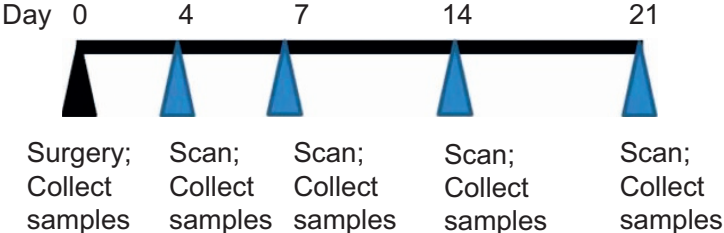

Figure 1. The strategy of skeletal injury. (a) A schematic diagram shows that $\mathrm{mT} / \mathrm{mG}$ is crossed to Ocn-Cre, and that Cre deletes tdTomato (red fluorescent) cassette and initiates GFP (green fluorescent) expression. (b) IHC was performed with anti-GFP antibody on distal femurs (upper figures) and midshafts (lower figures) from $\mathrm{mT} / \mathrm{mG}$ mice with (right figures, Ocn-CreTg/ + ) or without (left figures, Ocn-Cre $+/+$ ) Ocn-Cre; (c) A hole was generated at the anterior cortex of left tibia with a dental drill, and scanned by CT (in vivo) or micro-CT (ex vivo). (d) Time frame of the skeletal injury experiment. 


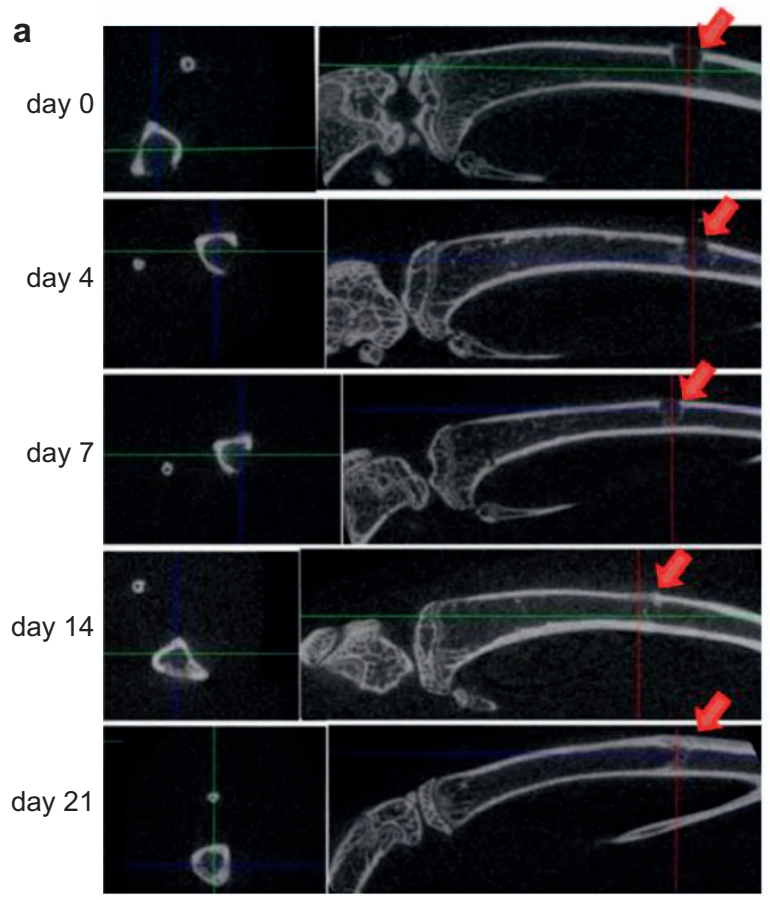

b

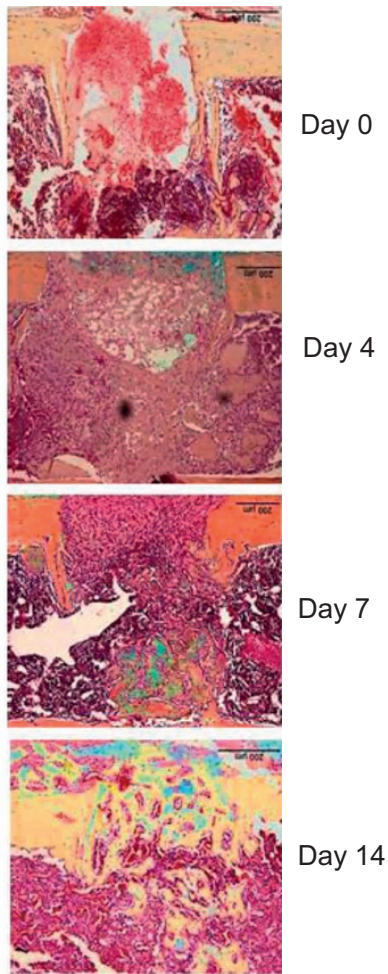

Figure 2. Early bone healing and mineralization are noted from 7 days post injury. (a) Transaxial 2-D images of injury sites and sagittal images of injured tibiae at different time points were taken with a micro-CT scanner ex vivo. Arrows indicate the injury sites; (b) Sagittal paraffin sections of the injured sites were stained with pentachrome. Bony tissue is in yellow/golden, and cartilage is bluish.

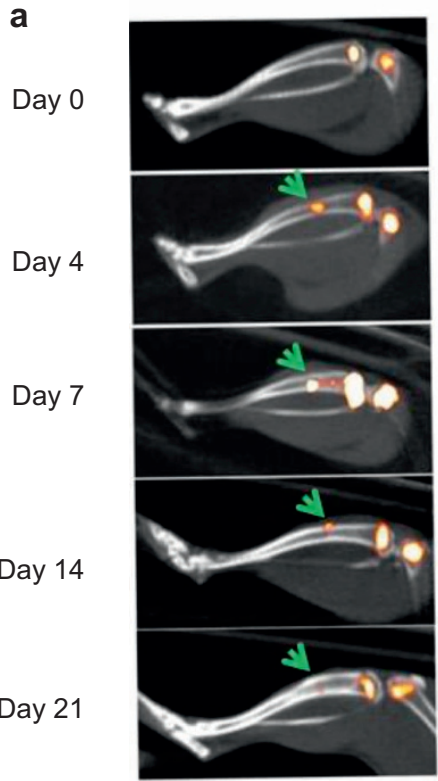

b

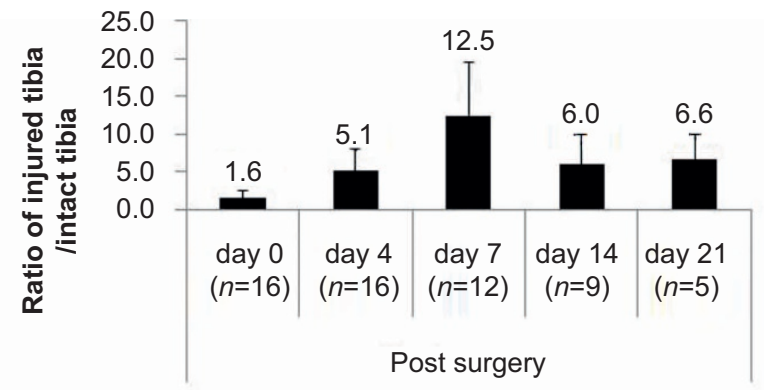

C

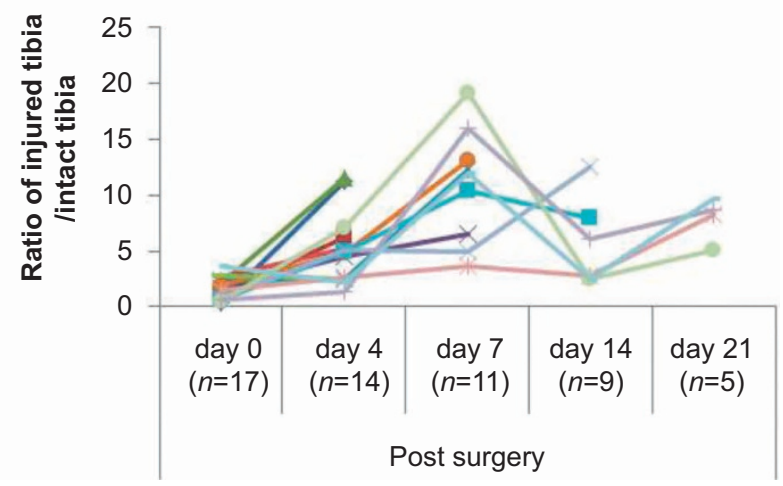

Figure 3. ${ }^{99 \mathrm{~m}} \mathrm{Tc}-\mathrm{MDP}$ uptake rate peaks at 7 days post injury. (a) Representative SPECT/CT images of injured tibias at each time point. Yellow pseudo color represents the intensity of ${ }^{99 \mathrm{~m}} \mathrm{Tc}-\mathrm{MDP}$ uptake. Note the growth-plate region in femurs and tibiae showed high ${ }^{99 \mathrm{~m}} \mathrm{Tc}-\mathrm{MD}$ intensity. The arrows indicate the injury sites; (b) Quantitated ${ }^{99 \mathrm{~m}} \mathrm{Tc}-\mathrm{MDP}$ uptake at the injury sites, which was normalized to the equivalent position of the intact tibia from the same animal. Each value is significantly changed $(P<0.05)$ compared with previous one except the last time point. $n$, mouse number used for SPECT/CT scans; (c) ${ }^{99 m}$ Tc-MDP uptake at the injury sites from each mouse throughout the healing process. Each line represents an individual animal. 
to form at the periosteum and endosteum adjacent to the injury sites 7 days post-surgery. Significant mineralized mass was present inside the injury sites 14 days post-surgery (Figure 2a). We then decalcified the tibia samples and stained the paraffin sections with pentachrome. Corresponding to the micro-CT data, we found bony tissue began to form in the cavity and cells populated the injury sites 7 days post-surgery (Figure 2b). We observed bony tissue filling the injury sites (Figure 2b), and tartrate-resistant acid phosphatase (TRAP) staining (associated with osteoclast activity) reached the highest level in the injury sites on day 14 post-surgery (Figure S1). These observations suggested that histologic data correlated well with the X-ray photographic data in monitoring bone healing.

Next we measured ${ }^{99} \mathrm{~m} T \mathrm{~T}-M D P$ uptake during bone healing. At each time point, we injected the injured mice with ${ }^{99 \mathrm{~m}} \mathrm{Tc}-\mathrm{MDP}$ and visualized isotope intensity in the injury sites by SPECT/CT scanning (Figure 3a). The relative isotope intensity in the injury site was normalized with the other intact tibia of the same animal. Although there was little mineralization noted in the injury sites 4 days post-surgery by micro-CT or histology (Figure $2 a$ and b), we observed threefold higher ${ }^{99 \mathrm{~m}}$ Tc-MDP uptake on day 4

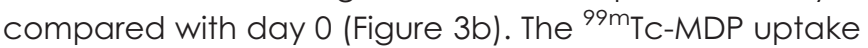
in the injury sites was highest on day 7 post-surgery, and decreased 14 and 21 days post-surgery (Figure 3b). The ${ }^{99 m}$ Tc-MDP-uptake dynamics in individual injured animals showed similar patterns (Figure 3c).

To determine how ${ }^{99 \mathrm{~m} T \mathrm{~T}-M D P-u p t a k e}$ dynamics correlate with osteoblast generation, we performed IHC analysis with anti-GFP antibody to identify mature osteoblasts. First, we focused on tissues adjacent to the injury sites. We found that the mature osteoblast population within the periosteal region increased robustly 7 days post-surgery, and chondrogenesis increased significantly on day 14 post-injury (Figure $4 a$ ). At the injury sites, the number of mature osteoblasts was increased at the cortical bone surface 4 days post-surgery (Figure 4b. There was high density of cells at the injury sites with a significant portion being mature osteoblasts, which were apparent on day 7 , and by 14 days

Day 0

Day 7

Day 14

a

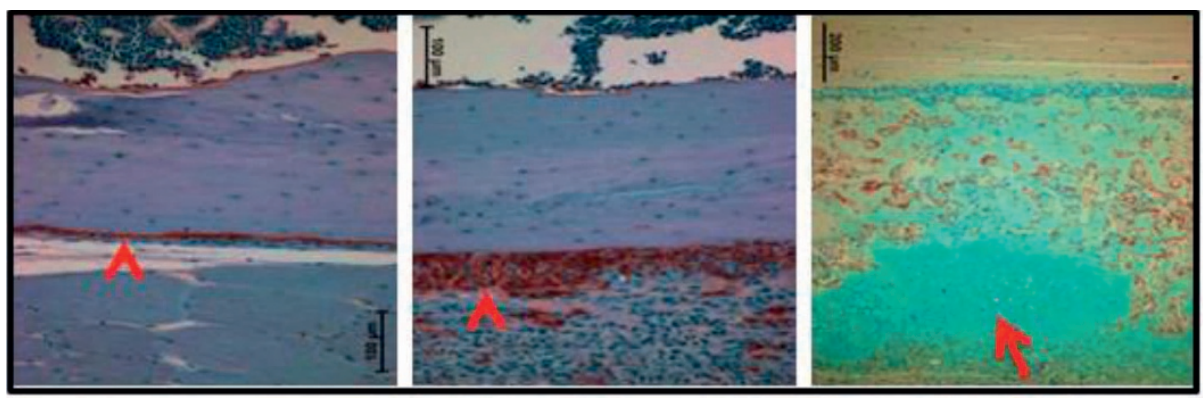

Day 4

Day 7

Day 14

b

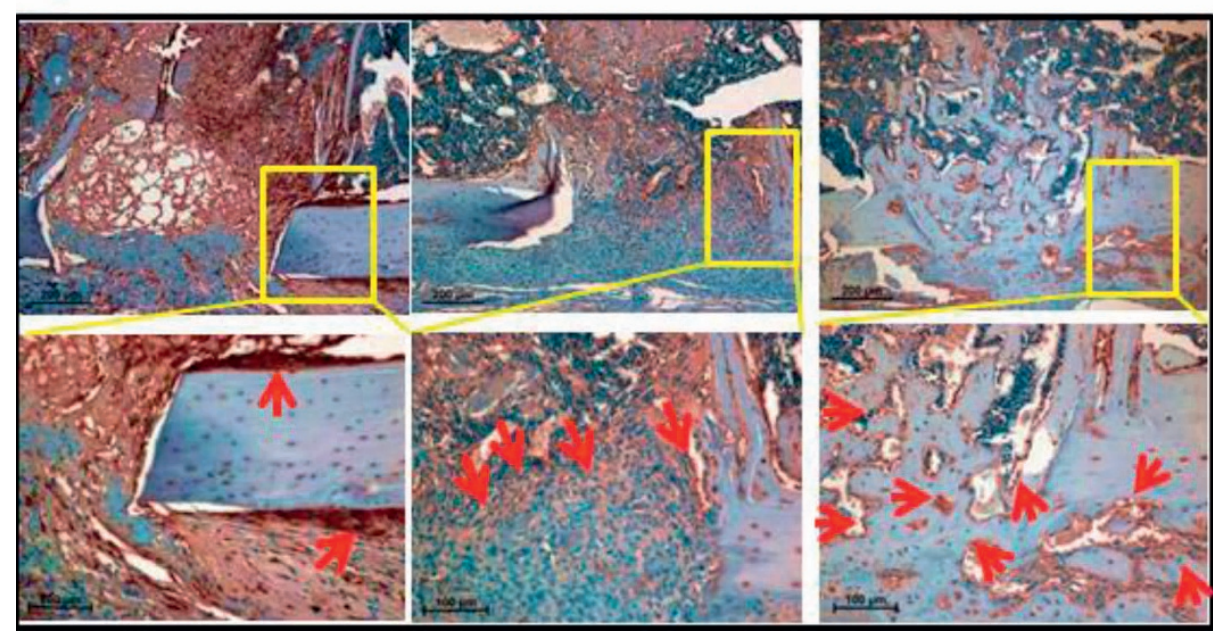

Figure 4. Osteoblast population increases significantly from 7 days post injury. (a) IHC analysis was performed with anti-GFP antibody on the injuryadjacent tissues. Arrowheads indicate osteoblasts at the periosteum region. The section at day 14 was co-stained with alcian blue, and the arrow indicates cartilage; (b) IHC was performed with anti-GFP antibody on the injury sites. Arrows indicate the GFP+ mature osteoblasts. The boxed regions are shown in higher magnifications. 
after surgery, most of the cells were mature osteoblasts (Figure 4b).

${ }^{99} \mathrm{~m}_{\text {TC-MDP }}$ uptake correlates to osteoblast differentiation and mineralization in vitro

We next isolated primary calvarial cells from 3-day-old Ocn-Cre:mT/mG neonates and differentiated the cells into osteoblasts with osteogenic medium. Using a fluorescent microscope, we observed that calvarial cells were differentiating into GFP+ mature osteoblasts (Figure 5a), which was confirmed by real-time PCR of Ocn expression (Figure 5b). The calvarial cells were pulsed with ${ }^{99} \mathrm{~m}$ Tc-MDP at different time points (day 0, 4, 7, 14, or 21 post-differentiation), and scanned by a planar gamma imager together with ${ }^{99} \mathrm{~m}$ Tc standards. We found that the ${ }^{99 \mathrm{~m}} \mathrm{Tc}-\mathrm{MDP}$ uptake increased with osteoblast marker Ocn expression (Figure $5 \mathrm{c}$ and $\mathrm{d}$ ). We further measured the ${ }^{99 \mathrm{~m}} \mathrm{Tc}-\mathrm{MDP}$ from the cell fraction collected by trypsinization and the residual mineral fraction dissolved in diluted hydrochloride. The ${ }^{99 \mathrm{~m}} \mathrm{Tc}-\mathrm{MDP}$ correlated with osteoblast maturation both in the cell fraction and in residual mineral fraction (Figure S2). We detected a small amount of calcium (by Alizarin red staining) and phosphate (by Von Kossa staining) in calvarial cells after 14 days of differentiation, and much higher levels of calcium and phosphate after 21 days of differentiation (Figure S3).

\section{DISCUSSION}

In this work, we investigated the accumulation of ${ }^{99} \mathrm{~m}$ Tc- MDP in skeletal injury sites with a specific emphasis on correlating the ${ }^{99 \mathrm{~m}}$ Tc-MDP accumulation with osteoblast differentiation. The result provided evidence that increased ${ }^{99 \mathrm{~m}}$ Tc-MDP uptake correlates with both osteoblast differentiation and mineralization. One caveat to note is that ${ }^{99 \mathrm{~m}}$ Tc-MDP uptake itself might have affected the bone repair process, which was not evaluated in the current study. The ${ }^{99 \mathrm{~m}}$ Tc-MDP uptake dynamics we observed in our skeletal injury model was similar to that found in previous studies on intraosseous implants, which showed that ${ }^{99 \mathrm{~m}} \mathrm{TC}$ MDP uptake occurred mostly during active bone formation and mineralization 7-10 days post-surgery in rats. ${ }^{13,25}$ A previous fracture-healing study showed that Ocn mRNA (as a mature osteoblast marker) increased from day 10, and peaked on day 15 post-fracture. ${ }^{26}$ We observed a similar a

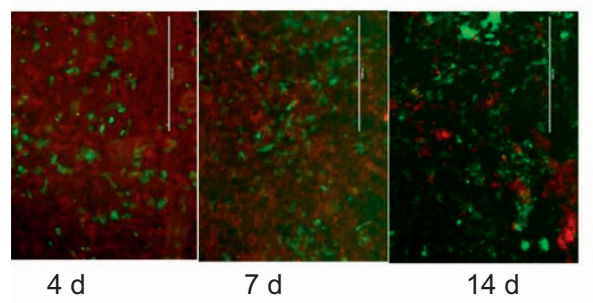

C

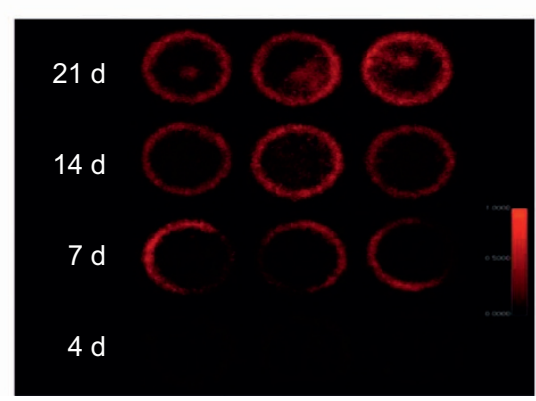

b
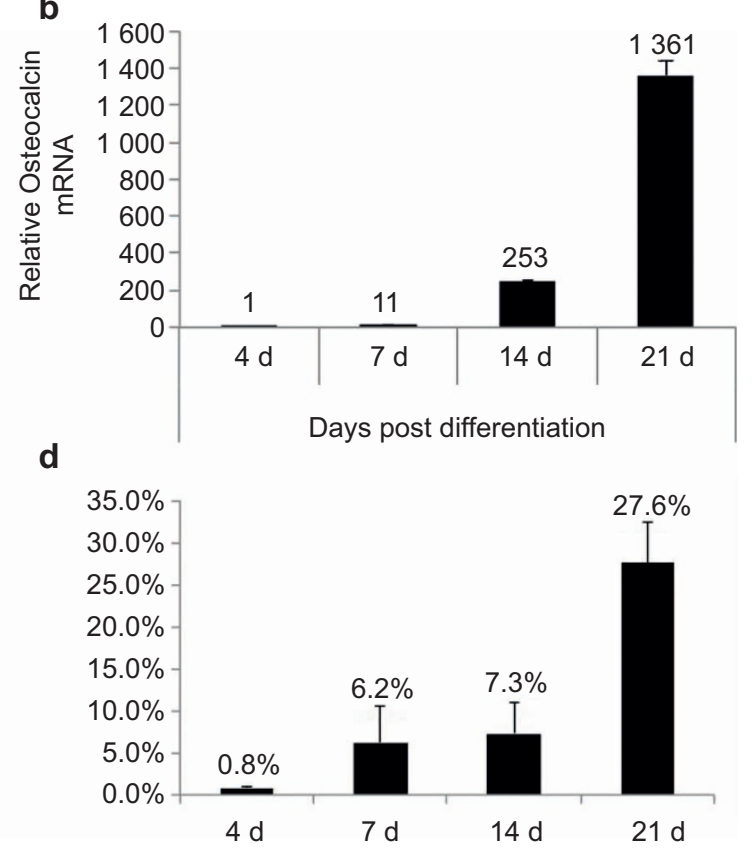

Days post differentiation

Figure 5. ${ }^{99 \mathrm{~m}} \mathrm{Tc}-\mathrm{MDP}$ uptake correlated with osteoblast differentiation in vitro. (a) Calvarial cells were isolated from Ocn-Cre; $m \mathrm{~T} / \mathrm{mG}$ neonates, differentiated into osteoblasts with osteogenic medium, and observed under a fluorescent microscopy. Green fluorescence indicates differentiated mature osteoblasts, and red fluorescence indicates all other non-osteoblast cells; (b) RNA was collected from differentiating osteoblasts at the indicated time points. Real-time PCR was performed to detect osteocalcin mRNA level normalized to the endogenous $\beta$-actin level; (c and d) Calvarial cells were plated on $30-\mathrm{cm}$ plates and differentiated with osteogenic media for the indicated time. Then cells were pulsed with ${ }^{99 \mathrm{~m}} \mathrm{Tc}-\mathrm{MDP}$ solution and visualized with a SPECT scanner. The actual images were taken with all the plates placed at the same layer and scanned at the same time (c). The quantitated ${ }^{99 \mathrm{~m}} \mathrm{Tc}-\mathrm{MDP}$ signal from each individual plate was normalized to total ${ }^{99 \mathrm{~m}} \mathrm{Tc}-\mathrm{MDP}$ signal from all plates (d). 
pattern of Ocn activation at protein level using the OcnCre:mT/mG model, in which Ocn is represented by GFP expression driven by the Ocn promoter (Figure 4b).

The nature of MDP phosphate has been generally neglected in bone-seeker research; it might be actively transported by cells, including osteoblasts. Evidence has shown that phosphate is a specific signal that modulates osteoblast differentiation by regulating protein function and gene expression. ${ }^{23,27-28}$ Osteoblasts actively gather phosphate through transporter mechanisms, which is important for primary calcification of the bone matrix. ${ }^{29-31}$ Recent studies using transmission electron microscopy verified the presence of nano-sized calcium phosphate within osteoblast mitochondrial granules and intracellular vesicles, suggesting that the storage and transport of such mineralcontaining vesicles may play important roles in mediating bone mineralization. ${ }^{32-33}$ Such evidence supports the possibility that ${ }^{99 \mathrm{~m}} \mathrm{Tc}-\mathrm{MDP}$ can also be captured and transported by osteoblasts through phosphate transporter/vesicles. In fact, bisphosphonates have direct biological functions on osteoblasts, in addition to previous recognition that they can affect the solubility of hydroxyapatite after being adsorbed. ${ }^{34-37}$

The ${ }^{99 \mathrm{~m}}$ Tc-MDP-uptake mechanism involving osteoblast differentiation that we proposed addresses the consideration of a balanced assessment of bone seeker uptake by organic and non-organic tissues. It will be informative for understanding the microscopic location of ${ }^{99 \mathrm{~m} T \mathrm{~T}-M D P}$ under physiological and pathological conditions.

\section{Author contributions:}

Zhendong A. Zhong, Tingtung A. Chang, and Bart $\mathrm{O}$. Williams conceived and designed the experiments; Zhendong A. Zhong, Anderson Peck, Shihong Li, Jeff VanOss, John Snider, and Casey J. Droscha performed research; Zhendong A. Zhong, Shihong Li, Tingtung A. Chang, and Bart O. Williams analyzed data; Zhendong A. Zhong and Shihong Li wrote the paper.

\section{Competing interests}

The authors declare no conflict of interest.

\section{Acknowledgements}

We are grateful to other members of the Williams Laboratory and the Program in Skeletal Disease and Tumor Metastasis for their comments and suggestions. This work is supported by the Van Andel Research Institute and by a grant to BOW from the NIH/NIAMS (AR053293). We also thank David Nadziejka for assistance in preparing the manuscript.

\section{References}

1 Love C, Din AS, Tomas MB, Kalapparambath TP, Palestro CJ. Radionuclide bone imaging: an illustrative review. Radiographics 2003; 23: $341-358$
2 Palma E, Correia JD, Campello MP, Santos I. Bisphosphonates as radionuclide carriers for imaging or systemic therapy. Mol Biosyst 2011; 7: 2950-2966

3 Even-Sapir E. Imaging of malignant bone involvement by morphologic, scintigraphic, and hybrid modalities. J Nucl Med 2005; 46: 1356-1367.

4 Peller PJ, Ho VB, Kransdorf MJ. Extraosseous Tc- $-{ }^{99 m}$ MDP uptake: a pathophysiologic approach. Radiographics 1993; 13: 715-734.

5 Kaye J, Hayward M. Soft tissue uptake on ${ }^{99 \mathrm{~m}} \mathrm{Tc}$ methylene diphosphonate bone scan imaging: pictorial review. Australas Radiol 2002; 46: 13-21.

6 Okamoto YM. Mechanism of accumulation of ${ }^{99 m} \mathrm{Tc}-M D P$ to bone: correlation of in vivo data with in vitro data. Radiat Med 1997; 15: 209-215.

7 Seto H, Ihara F, Futatsuya $\mathrm{R}$ et al. Simultaneous measurements of to]twenty-four-hour whole-body retention of $47 \mathrm{Ca}$-chloride and ${ }^{99 \mathrm{~m}} \mathrm{Tc}-$ MDP: early differentiation of metabolic bone diseases in rat models. Nucl Med Biol 1993; 20: 337-342.

8 Toegel S, Hoffmann O, Wadsak W et al. Uptake of bone-seekers is solely associated with mineralisation! A study with ${ }^{99 \mathrm{~m}} \mathrm{Tc}-\mathrm{MDP}, 153 \mathrm{Sm}-$ EDTMP and 18F-fluoride on osteoblasts. Eur J Nucl Med Mol Imaging 2006; 33: 491-494.

9 Leitha T, Staudenherz A, Scholz U. Three-phase bone scintigraphy of hydroxyapatite ocular implants. Eur J Nucl Med 1995; 22: 308-314.

10 Kanishi D. ${ }^{99 \mathrm{~m}} \mathrm{Tc}-\mathrm{MDP}$ accumulation mechanisms in bone. Oral Surg Oral Med Oral Pathol 1993; 75: 239-246.

11 Mitterhauser M, Toegel S, Wadsak W et al. Binding studies of [18F]fluoride and polyphosphonates radiolabelled with [99mTc], [111In], [153Sm] and [188Re] on bone compartments: verification of the pre vivo model? Bone 2005; 37: 404-412.

12 Einhorn TA, Vigorita VJ, Aaron A. Localization of technetium. $-{ }^{99 \mathrm{~m}}$ methylene diphosphonate in bone using microautoradiography. J Orthop Res 1986; 4: 180-187.

13 Schwartz Z, Shani J, Soskolne WA, Touma H, Amir D , Sela J. Uptake and biodistribution of technetium-99m-MD32P during rat tibial bone repair. $J$ Nucl Med 1993; 34: 104-108.

14 Sela J, Gross UM, Kohavi D et al. Primary mineralization at the surfaces of implants. Crit Rev Oral Biol Med 2000; 11: 423-436.

15 Kaye M, Silverton S, Rosenthall L. Technetium-99m-pyrophosphate: studies in vivo and in vitro. J Nucl Med 1975; 16: 40-45.

16 Rosenthall L, Kaye M. Observations on the mechanism of ${ }^{99 \mathrm{~m}}$ Tc-labeled phosphate complex uptake in metabolic bone disease. Semin Nucl Med 1976; 6: 59-67.

17 Zimmer AM, Isitman AT, Holmes RA. Enzymatic inhibition of diphosphonate: a proposed mechanism of tissue uptake. J Nucl Med 1975; 16: 352-356.

18 Christensen SB, Arnold CC. Distribution of ${ }^{99 \mathrm{~m}} \mathrm{Tc}-$ phosphate compounds in osteoarthritic femoral heads. J Bone Joint Surg Am 1980; 62: 90-96.

19 Bonewald LF. The amazing osteocyte. J Bone Miner Res 2011; 26: 229-238.

20 Zhang X, Awad HA, O'Keefe RJ, Guldberg RE, Schwarz EM. A perspective: engineering periosteum for structural bone graft healing. Clin Orthop Relat Res 2008; 466: 1777-1787.

21 Zhang M, Xuan S, Bouxsein ML et al. Osteoblast-specific knockout of the insulin-like growth factor (IGF) receptor gene reveals an essential role of IGF signaling in bone matrix mineralization. J Biol Chem 2002; 277: 44005-44012.

22 Muzumdar MD, Tasic B, Miyamichi K, Li L, Luo L. A global doublefluorescent Cre reporter mouse. Genesis 2007; 45: 593-605.

23 Beck GR Jr, Zerler B, Moran E. Phosphate is a specific signal for induction of osteopontin gene expression. Proc Natl Acad Sci U S A 2000; 97: 8352-8357. 
24 Zhong Z, Zylstra-Diegel CR, Schumacher CA et al. Wntless functions in mature osteoblasts to regulate bone mass. Proc Natl Acad Sci U S A 2012; 109: E2197-E2204.

25 Sela J, Shani J, Kohavi D et al. Uptake and biodistribution of ${ }^{99} \mathrm{~m}_{\text {technetium methylene-[32P] diphosphonate during endosteal }}$ healing around titanium, stainless steel and hydroxyapatite implants in rat tibial bone. Biomaterials 1995; 16: 1373-1380.

26 Jingushi S, Joyce ME, Bolander ME. Genetic expression of extracellular matrix proteins correlates with histologic changes during fracture repair. J Bone Miner Res 1992; 7: 1045-1055.

27 Beck GR, Jr. Inorganic phosphate as a signaling molecule in osteoblast differentiation. J Cell Biochem 2003; 90: 234-243.

28 Khoshniat S, Bourgine A, Julien M, Weiss P, Guicheux J, Beck L. The emergence of phosphate as a specific signaling molecule in bone and other cell types in mammals. Cell Mol Life Sci 2011; 68: 205-218.

29 Floege J, Ketteler M. Vascular calcification in patients with end-stage renal disease. Nephrol Dial Transplant 2004; 19(Suppl 5) : V59-V66.

30 Suzuki A, Ghayor C, Guicheux J et al. Enhanced expression of the inorganic phosphate transporter Pit-1 is involved in BMP-2-induced matrix mineralization in osteoblast-like cells. J Bone Miner Res 2006; 21: 674-683.

31 Lundquist P, Murer H, Biber J. Type II Na+-Pi cotransporters in osteoblast mineral formation: regulation by inorganic phosphate. Cell Physiol Biochem 2007; 19: 43-56.

32 Mahamid J, Sharir A, Gur D, Zelzer E, Addadi L, Weiner S. Bone mineralization proceeds through intracellular calcium phosphate loaded vesicles: a cryo-electron microscopy study. J Struct Biol 2011; 174: 527-535.

33 Boonrungsiman S, Gentleman E, Carzaniga $\mathrm{R}$ et al. The role of intracellular calcium phosphate in osteoblast-mediated bone apatite formation. Proc Natl Acad Sci U S A 2012; 109: 14170-14175.

34 Cornish J, Bava U, Callon KE, Bai J, Naot D, Reid IR. Bone-bound bisphosphonate inhibits growth of adjacent non-bone cells. Bone 2011; 49: 710-716.

35 Plotkin LI, Weinstein RS, Parfitt AM, Roberson PK, Manolagas SC, Bellido T. Prevention of osteocyte and osteoblast apoptosis by bisphosphonates and calcitonin. J Clin Invest 1999; 104: 1363-1374.

36 Idris AI, Rojas J, Greig IR, Van't Hof RJ, Ralston SH. Aminobisphosphonates cause osteoblast apoptosis and inhibit bone nodule formation in vitro. Calcif Tissue Int 2008; 82: 191-201.

37 Iwata K, Li J, Follet H, Phipps RJ, Burr DB. Bisphosphonates suppress periosteal osteoblast activity independently of resorption in rat femur and tibia. Bone 2006; 39: 1053-1058.

(c) (i) $\odot$ This work is licensed under a Creative Commons AttributionGy NC ND Nommercial-NoDerivs 3.0 Unported License. The images or other third party material in this article are included in the article's Creative Commons license, unless indicated otherwise in the credit line; if the material is not included under the Creative Commons license, users will need to obtain permission from the license holder to reproduce the material. To view a copy of this license, visit http://creativecommons.org/licenses/by-nc-nd/3.0/

Supplemental Information for this article can be found on the Bone Research website (http://www.nature.com/boneres). 